\title{
Survival estimation for singly type one censored sample based on generalized Rayleigh distribution
}

\author{
Iden H. Alkanani* Hind J. Kadhum**
}

Received 20, December, 2012

Accepted 12, June, 2013

\begin{abstract}
:
This paper interest to estimation the unknown parameters for generalized Rayleigh distribution model based on censored samples of singly type one. In this paper the probability density function for generalized Rayleigh is defined with its properties . The maximum likelihood estimator method is used to derive the point estimation for all unknown parameters based on iterative method, as Newton Raphson method, then derive confidence interval estimation which based on Fisher information matrix . Finally, testing whether the current model (GRD) fits to a set of real data, then compute the survival function and hazard function for this real data.
\end{abstract}

Key words: Maximum likelihood method, type one censored sample, interval estimation method, and fisher information matrix .

\section{1: Introduction:}

A rich class of probability distributions had been introduced by Burr ( 1942 ), which includes twelve different form of cumulative distribution functions for modeling data ${ }^{[1]}$.

Rayleigh distribution is an important distribution in statistics and operations research. It has a wide range of applications in several areas such as health, agriculture , biology , analyzing wind speed data and other sciences ${ }^{[2],[3]}$.

It is known that the generalized Rayleigh density functions are always right skewed and they can be used quite effectively to analyze skewed data set .

Recently, Surles and Padgett (2001) introduced two parameters Burr-Type X distribution, which can also be described as generalized Rayleigh distribution (G.R.D)and it was observed that this particular

skewed distribution can be used in analyzing lifetime data ${ }^{[7]}$.

Kundu and Raqab ; (2003) consider this distribution and discussed its different properties and employed different methods of estimators ${ }^{[4]}$.

Kundu and Raqab ; (2005) ${ }^{[4]}$ estimate the parameters of (G.R.D) by using different methods, such as MLE method modified moment method, percentiles method, least square method .

Kundu and Raqab ; (2006) presented that the pdf of a (G.R.D) is a decreasing function for $\alpha \leq 0.5$ and it is a right - skewed unimodal function for $\alpha>0.5$. ( Figure 1$)^{[6]}$.

And show that the hazard rate function of a (G.R.D) is bathtub type for $\alpha \leq 0.5$ and it is an increasing function for $\alpha>0.5$. ( Figure 2$)^{[6]}$.

*University of Baghdad, College of science for women, Department of Mathematics 

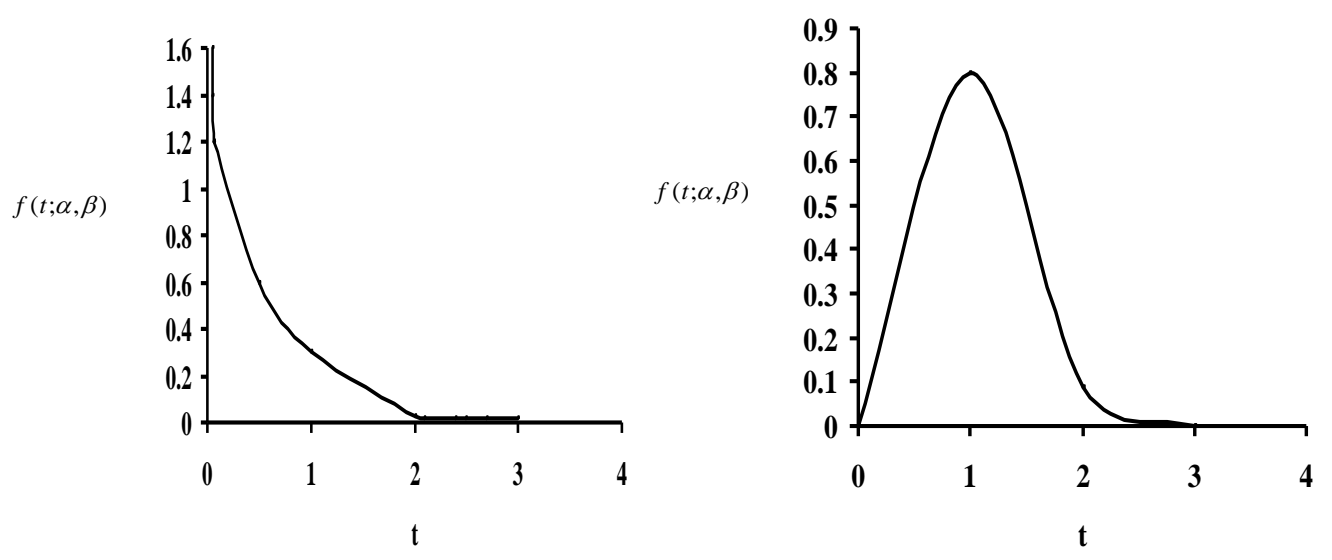

Left $\alpha=0.25 ; \beta=1$ Figure (1)Right $\alpha=0.25 ; \beta=1$
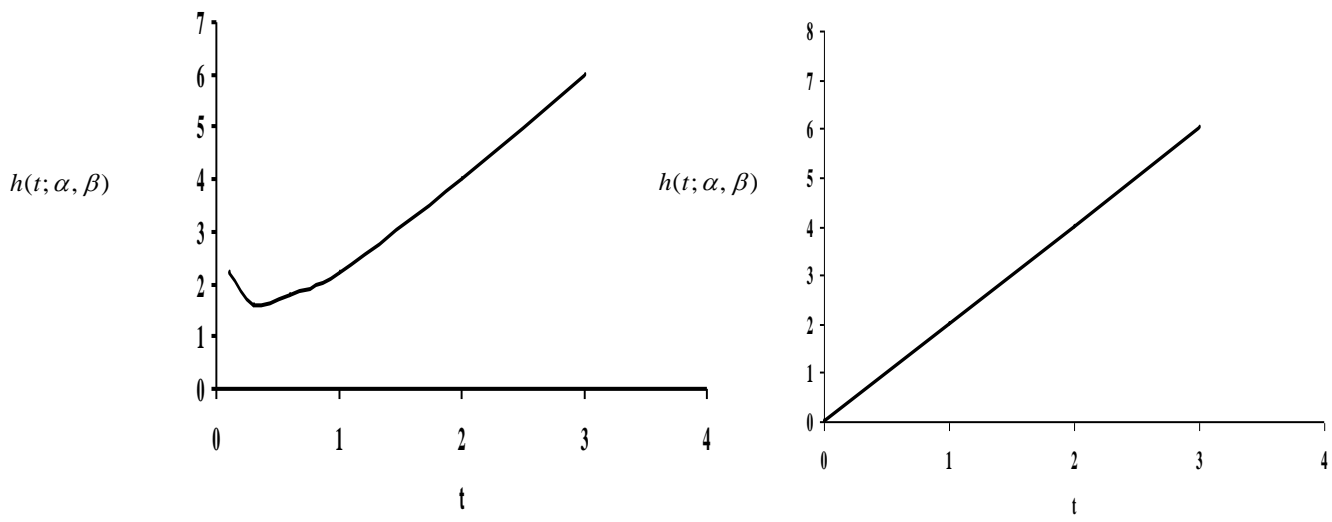

Left $\alpha=0.25 ; \beta=1$ Figure (2)Right $\alpha=1 ; \beta=1$

Our aim in this paper is to estimate the unknown parameters of the (G.R.D) based on censored data .

The maximum likelihood method is used to derive point estimation by using Newton-Raphson method, then derive and find the confidence interval estimation based on Fisher information matrix .

After that deriving and estimating the survival function and hazard function, then fit and apply this distribution to a set of real data to calculate the values of estimators and compute the values of estimate for survival and hazard function .

The rest of the paper is organized as follows : in section two definition and some properties of distribution, in section three deriving point estimation, in section four deriving interval estimation for the unknown parameter of study distribution, in section five apply the real set data then compute the estimators, survival function and hazard function. Finally, in section six made conclusion for this paper .

\section{2: Definition and properties ${ }^{[1]}$}

The probability density function for generalized Rayleigh distribution (G.R.D) is :

$f_{G R}(t ; \alpha, \beta)=\left\{\begin{array}{lr}2 \alpha \beta t e^{-\beta t^{2}}\left(1-e^{-\beta t^{2}}\right)^{\alpha-1} & t \geq 0 \\ 0 & \text { o.w }\end{array} \ldots . .\right.$. (l)

$\Omega=\{(\alpha, \beta) ; \alpha>0, \beta>0\}$

Where $\beta$ : is shape parameter

$\alpha$ : is scale parameter 
From now on the generalized Rayleigh distribution with the scale parameter $\alpha$ and shape parameter $\beta$ will be denoted by $\operatorname{GR}(\alpha, \beta)$.

The cumulative distribution function for this distribution is :

$F(t ; \alpha, \beta)=\left(1-e^{-\beta t^{2}}\right)^{\alpha} \quad ; \quad$ for $t>0$

It's survival function is given by :

$$
\begin{aligned}
& S(t ; \alpha, \beta)=1-\left(1-e^{-\beta t^{2}}\right)^{\alpha} \quad ; \quad \text { for } \\
& t \geq 0 \ldots \ldots . .(\beta)
\end{aligned}
$$

The hazard rate function is given by :

$$
h(t ; \alpha, \beta)=\frac{2 \alpha \beta t e^{-\beta t^{2}}\left(1-e^{-\beta t^{2}}\right)^{\alpha-1}}{1-\left(1-e^{-\beta t^{2}}\right)^{\alpha}} ;
$$

for $t \geq 0$

\section{3:Maximum}

likelihood

\section{estimator method for censored}

\section{type one sample}

The maximum likelihood method is the most popular procedure to estimate the parameter $\theta$ which specifies a probability function $f(t: \theta)$, based on the observations $t_{1}, t_{2}, \ldots . ., t_{n}$ which were independently sample from the distribution .

The likelihood function of type I censored data is :

$$
\begin{aligned}
& L=\frac{n !}{(n-r) !}\left[\prod_{i=1}^{r} f\left(t_{i}\right)\right]\left[1-F\left(T_{0}\right)\right]^{n-r} \\
& 0 \leq t_{(1)} \leq t_{(2)} \leq \ldots . . \leq t_{(m)} \leq t_{(0)} \ldots \ldots \ldots \ldots . . .(5) \\
& \text { Let } \frac{n !}{(n-r) !}=a \\
& \left.L=a\left[\prod_{i=1}^{r} 2 \alpha \beta t_{i} e^{-\beta t_{i}^{2}}\left(1-e^{-\beta t_{i}^{2}}\right)^{\alpha-1}\right]\left[1-\left(1-e^{-\beta t_{0}^{2}}\right)^{\alpha}\right)\right]^{n-r} \\
& L=a 2^{r} \alpha^{r} \beta^{r} \prod_{i=1}^{r} t_{i} e^{-\beta \sum_{i=1}^{r} t_{i}^{2}} \prod_{i=1}^{r}\left(1-e^{-\beta t_{i}^{2}}\right) \\
& \left.{ }^{\alpha-1}\left[1-\left(1-e^{-\beta t_{0}^{2}}\right)^{\alpha}\right)\right]^{n-r} \ldots . . .(6)
\end{aligned}
$$

Taking the logarithm for the likelihood function, so we get the function :

$$
\ln L=\ln a+r \ln 2+r \ln \alpha+r \ln \beta+
$$$$
\sum_{i=1}^{r} \ln t_{i}-\beta \sum_{i=1}^{r} t_{i}^{2}+(\alpha-1) \sum_{i=1}^{r} \ln \left(1-e^{-\beta t_{i}^{2}}\right)
$$$$
\left.+(n-r) \ln \left[1-\left(1-e^{-\beta t_{0}^{2}}\right)^{\alpha}\right)\right]
$$

The partial derivatives for the $\log -$ likelihood function with respect to unknown parameters $\alpha$ and $\beta$ are :

$$
\begin{aligned}
& \frac{\partial \ln L}{\partial \alpha}=\frac{r}{\alpha}+\sum_{i=1}^{r} \ln \left(1-e^{-\beta t_{i}^{2}}\right)- \\
& \frac{(n-r)\left(1-e^{-\beta t_{0}^{2}}\right)^{\alpha} \ln \left(1-e^{-\beta t_{0}^{2}}\right)}{1-\left(1-e^{-\beta t_{0}^{2}}\right)^{\alpha}}
\end{aligned}
$$

We place the partial derivative for $\alpha$ to zero as follows :

$\frac{\partial \ln L}{\partial \alpha}=0$

$$
\begin{gathered}
\frac{r}{\hat{\alpha}}+\sum_{i=1}^{r} \ln \left(1-e^{-\hat{\beta} t_{i}^{2}}\right)-\frac{(n-r)\left(1-e^{-\hat{\beta} t_{0}^{2}}\right)^{\hat{\alpha}} \ln \left(1-e^{-\hat{\beta} t_{0}^{2}}\right)}{1-\left(1-e^{-\hat{\beta} t_{0}^{2}}\right)^{\hat{\alpha}}}=0 \ldots \ldots \ldots \ldots \ldots \ldots \ldots \ldots \\
\hat{\alpha}=\frac{r\left[1-\left(1-e^{-\hat{\beta} T_{0}^{2}}\right)^{\hat{\alpha}}\right]}{\left(1-e^{-\hat{\beta} T_{0}^{2}}\right)^{\hat{\alpha}}\left[(n-r) \ln \left(1-e^{-\hat{\beta} T_{0}^{2}}\right)+\sum_{i=1}^{r} \ln \left(1-e^{-\hat{\beta} t_{i}^{2}}\right)\right]-\sum_{i=1}^{r} \ln \left(1-e^{-\hat{\beta} t_{i}^{2}}\right)} \\
\frac{\partial \operatorname{lm} L}{\partial \beta}=\frac{r}{\beta}-\sum_{i=1}^{r} t_{i}^{2}+(\alpha-1) \sum_{i=1}^{r} \frac{t_{i}^{2} e^{-\beta t_{i}^{2}}}{1-e^{-\beta t_{i}^{2}}}-\frac{(n-r) \alpha T_{0}^{2} e^{-\beta T_{0}^{2}}\left(1-e^{-\beta T_{0}^{2}}\right)^{\alpha-1}}{1-\left(1-e^{-\beta T_{0}^{2}}\right)^{\alpha}}
\end{gathered}
$$

Equating the partial derivative for $\beta$ to zero and we solve this equation : 


$$
\begin{gathered}
\frac{\partial \ln L}{\partial \beta}=0 \\
\frac{r}{\hat{\beta}}-\sum_{i=1}^{r} t_{i}^{2}+(\hat{\alpha}-1) \sum_{i=1}^{r} \frac{t_{i}^{2} e^{-\hat{\beta} t_{i}^{2}}}{1-e^{-\hat{\beta} t_{i}^{2}}}-\frac{(n-r) \hat{\alpha} T_{0}^{2} e^{-\hat{\beta} T_{0}^{2}}\left(1-e^{-\hat{\beta} T_{0}^{2}}\right)^{\hat{\alpha}-1}}{1-\left(1-e^{-\hat{\beta} T_{0}^{2}}\right)^{\hat{\alpha}}}=0 \\
\hat{\beta}=\frac{r}{\sum_{i=1}^{r} t_{i}^{2}-(\hat{\alpha}-1) \sum_{i=1}^{r} \frac{t_{i}^{2} e^{-\hat{\beta} t_{i}^{2}}}{1-e^{-\hat{\beta} t_{i}^{2}}}+\frac{(n-r) \hat{\alpha} T_{0}^{2} e^{-\hat{\beta} T_{0}^{2}}\left(1-e^{-\hat{\beta} T_{0}^{2}}\right)^{\hat{\alpha}-1}}{1-\left(1-e^{-\hat{\beta} T_{0}^{2}}\right)^{\hat{\alpha}}}} \ldots . . . . .
\end{gathered}
$$

Since we cannot find the estimators for the parameters $(\alpha, \beta)$, because hardness solve this nonlinear equations simultaneously, so we resort to iterative methods in numerical analysis .

Consider Newton-Raphson method ${ }^{[2]}$ is one of the best iterative methods in numerical analysis because it's very fast and the error of this iterative method is quadratic approximation . An iterative procedure is a technique of successive approximations , and each approximation is called iteration .

If the successive approximations approach the solution very closely then the iterations converge .

The Newton - Raphson method requires an initial value of each unknown parameters $(\alpha, \beta)$.

The steps of this method are as follows

$$
\left[\begin{array}{l}
\alpha_{k+1} \\
\beta_{k+1}
\end{array}\right]=\left[\begin{array}{l}
\alpha_{k} \\
\beta_{k}
\end{array}\right]-J_{k}^{-1}\left[\begin{array}{l}
f_{1}(\alpha) \\
f_{2}(\beta)
\end{array}\right]
$$

The two - functions $f_{1}(\alpha)$ and $f_{2}(\beta)$ are the first derivative of $\log$ likelihood function with respect to unknown parameters $\alpha$ and $\beta$ respectively .

$$
\begin{gathered}
f_{1}(\alpha)=\frac{r}{\alpha}+\sum_{i=1}^{r} \ln \left(1-e^{-\beta t_{i}^{2}}\right)-\frac{(n-r)\left(1-e^{-\beta T_{0}^{2}}\right)^{\alpha} \ln \left(1-e^{-\beta T_{0}^{2}}\right)}{1-\left(1-e^{-\beta T_{0}^{2}}\right)^{\alpha}} \ldots \ldots . . . . . \\
f_{2}(\beta)=\frac{r}{\beta}-\sum_{i=1}^{r} t_{i}^{2}+(\alpha-1) \sum_{i=1}^{r} \frac{t_{i}^{2} e^{-\beta t_{i}^{2}}}{1-e^{-\beta t_{i}^{2}}}-\frac{(n-r) \alpha T_{o}^{2} e^{-\beta T_{0}^{2}}\left(1-e^{-\beta T_{0}^{2}}\right)^{\alpha-1}}{1-\left(1-e^{-\beta T_{0}^{2}}\right)^{\alpha}}
\end{gathered}
$$

The Jacobean matrix $J_{k}$ is the first derivative for each function of $f_{1}(\alpha)$ and $f_{2}(\beta)$ are with respect to $\alpha$ and

$$
J_{k}=\left[\begin{array}{ll}
\frac{\partial f_{1}(\alpha)}{\partial \alpha} & \frac{\partial f_{1}(\alpha)}{\partial \beta} \\
\frac{\partial f_{2}(\beta)}{\partial \alpha} & \frac{\partial f_{2}(\beta)}{\partial \beta}
\end{array}\right]=\left[\begin{array}{l}
\frac{\partial^{2} \ln L}{\partial \alpha^{2}} \\
\frac{\partial^{2} \ln L}{\partial \beta \partial \alpha}
\end{array}\right.
$$

Then : $\beta$ or it is the second derivative of the log-likelihood function to the two parameters .

$$
\left.\begin{array}{c}
\frac{\partial^{2} \ln L f_{1}(\alpha)}{\partial \alpha \partial \beta} \\
\frac{\partial^{2} \ln L}{\partial \beta^{2}}
\end{array}\right]
$$




$$
\begin{aligned}
& \frac{\partial f_{1}(\alpha)}{\partial \alpha}=-\frac{r}{\alpha^{2}}-\frac{(n-r)\left(1-e^{-\beta T_{0}^{2}}\right)^{\alpha}\left[\ln \left(1-e^{-\beta T_{0}^{2}}\right)\right]^{2}}{\left[1-\left(1-e^{-\beta t_{0}^{2}}\right)^{\alpha}\right]^{2}} \\
& \frac{\partial f_{1}(\alpha)}{\partial \beta}=\sum_{i=1}^{r} \frac{t_{i}^{2} e^{-\beta t_{i}^{2}}}{1-e^{-\beta t_{i}^{2}}}-\frac{(n-r) T_{o}^{2} e^{-\beta T_{0}^{2}}\left(1-e^{-\beta T_{0}^{2}}\right)^{\alpha-1}\left[1+\alpha \ln \left(1-e^{-\beta T_{0}^{2}}\right)-\left(1-e^{-\beta T_{0}^{2}}\right)^{\alpha}\right]}{\left[1-\left(1-e^{-\beta T_{0}^{2}}\right)^{\alpha}\right]^{2}} \\
& \frac{\partial f_{2}(\beta)}{\partial \alpha}=\sum_{i=1}^{r} \frac{t_{i}^{2} e^{-\beta t_{i}^{2}}}{1-e^{-\beta t_{i}^{2}}}-\frac{(n-r) T_{o}^{2} e^{-\beta T_{0}^{2}}\left(1-e^{-\beta T_{0}^{2}}\right)^{\alpha-1}\left[1+\alpha \ln \left(1-e^{-\beta T_{0}^{2}}\right)-\left(1-e^{-\beta T_{0}^{2}}\right)^{\alpha}\right]}{\left[1-\left(1-e^{-\beta T_{0}^{2}}\right)^{\alpha}\right]^{2}} \\
& \frac{\partial f_{2}(\beta)}{\partial \beta}=-\frac{r}{\beta^{2}}-(\alpha-1) \sum_{i=1}^{r} \frac{t_{i}^{2} e^{-\beta t_{i}^{2}}}{\left(1-e^{-\beta t_{i}^{2}}\right)^{2}} \\
& -\frac{(n-r) \alpha T_{0}^{4} e^{-\beta T_{0}^{2}}\left(1-e^{-\beta T_{0}^{2}}\right)^{\alpha-1}\left[\frac{(\alpha-1) e^{-\beta T_{0}^{2}}}{1-e^{-\beta T_{0}^{2}}}-1+\left(1-e^{-\beta T_{0}^{2}}\right)^{\alpha}+e^{-\beta T_{0}^{2}}\left(1-e^{-\beta T_{0}^{2}}\right)^{\alpha-1}\right]}{\left[1-\left(1-e^{-\beta T_{0}^{2}}\right)^{\alpha}\right]^{2}}
\end{aligned}
$$

the Jacobean matrix in maximum likelihood method estimator must be anon-singular symmetric matrix in this procedure because depending upon the first derivatives, so it's inverse can be founded .

The absolute value for the difference between the new founded values with the initial value is the error term, it must be a symbol by $\varepsilon$, which is a very small value and assumed .

Then, error term is formulated as :

$\left[\begin{array}{c}\varepsilon_{k+1}(\alpha) \\ \varepsilon_{k+1}(\beta)\end{array}\right]=\left\lfloor\left[\begin{array}{c}\alpha_{k+1} \\ \beta_{k+1}\end{array}\right]-\left[\begin{array}{c}\alpha_{k} \\ \beta_{k}\end{array}\right] \mid\right.$

\section{4:Interval estimation method}

The interval estimation is a range of estimation, where the true value of parameter lies between lower limit estimate and upper limit estimate - The measure which represent the interval estimation is confidence intervals, which is a range of values, bounded above and below, within which the true unknown value is expected to fall, that means, confidence intervals are estimates of the true unknown parameters. The confidence bounds for the shape and scale parameters in the generalized Rayleigh distribution are as follows :

$$
\begin{aligned}
& \hat{\alpha}_{\text {Lower }}=\hat{\alpha}-Z_{\alpha} \sqrt{\operatorname{var}(\hat{\alpha})} \\
& \hat{\alpha}_{\text {Upper }}=\hat{\alpha}-Z_{\alpha} \sqrt{\operatorname{var(\hat {\alpha })}} \\
& \hat{\beta}_{\text {Lower }}=\hat{\beta}-Z_{\alpha} \sqrt{\operatorname{var}(\hat{\beta})} \\
& \hat{\beta}_{\text {Upper }}=\hat{\beta}-Z_{\alpha} \sqrt{\operatorname{var}(\hat{\beta})}
\end{aligned}
$$

Where $Z_{\alpha}$ is tabulate value of standard normal distribution under level of significant $\alpha$. Now, to find $\operatorname{var}(\alpha)$ and $\operatorname{var}(\beta)$ we must employ the observed fisher information matrix , where the variance-covariance matrix can be obtained by inverting the fisher information matrix, which are as follows :

$$
I(\alpha, \beta)=\left[\begin{array}{cc}
-\frac{\partial f_{1}(\alpha)}{\partial \alpha} & -\frac{\partial f_{1}(\alpha)}{\partial \beta} \\
-\frac{\partial f_{2}(\beta)}{\partial \alpha} & -\frac{\partial f_{2}(\beta)}{\partial \beta}
\end{array}\right]^{-1}=
$$

$\left[\begin{array}{cc}\operatorname{var} \hat{\alpha}) & \operatorname{cov}(\hat{\alpha}, \hat{\beta}) \\ \operatorname{cov} \hat{\alpha}, \hat{\beta}) & \\ \operatorname{var}(\hat{\beta})\end{array}\right] \ldots . .(27)$

After that, we can find the confidence interval estimation for the 
survival function and hazard function which are formulated as follows :

$$
\begin{aligned}
& s(t)=1-\left(1-e^{-\hat{\beta} t_{i}^{2}}\right)^{\hat{\alpha}} \ldots \ldots \ldots \ldots . . . . \\
& h(t)=\frac{2 \hat{\alpha} \hat{\beta} t_{i} e^{-\hat{\beta} t_{i}^{2}}\left(1-e^{-\hat{\beta} t_{i}^{2}}\right)^{\hat{\alpha-1}}}{1-\left(1-e^{-\hat{\beta} t_{i}^{2}}\right)^{\hat{\alpha}}}
\end{aligned}
$$

\section{5: results and discussion}

In this paper, depending on real data for the Lung cancer disease , choosing this type of cancer because it is widespread and deadly in time in Iraq and this type of diseases has failure time ( death time) occurs which is interesting phenomenon in this paper .

To collect data for the Lungcancer disease, returning the educational hospital in Diwaniya .

The time of study point in this paper determined from 1-1-2012 until 31-12-2012, that means the duration time of this study is constant and fixed for (12) months or (365) days .

The number of patients in the experiment for the above duration time is (60) . Thirty seven patients were dead and twenty three patients remain alive .

We found from the our samples that the Male formed (51) and Female formed (31) from my study. And, we found that Urban is (59) and Rural (23) for my study .

When applying the test statistic (Kolmogorov-Smirnov) in order to fit generalized Rayleigh distribution data , it is discovered that the calculated value is $(0.20833)$; when comparing this value with tabulated value (0.2183) we find out that the calculated value is less than the tabulated value at level of significant $(0.05)$, this means data is distributed according to generalized Rayleigh distribution .

The null and alternative hypotheses are as follows :

$H_{0}$ : The survival time data is distributed as generalized Rayleigh pdf

$H_{1}$ : The survival time data is not distributed as generalized Rayleigh pdf

By utilizing program visual studio c++, we've got the following estimated parameters values :

$\hat{\alpha}=0.00465 \quad ; \hat{\beta}=0.01281$

Now, we find and calculated the variance / covariance matrix by using observed Fisher information matrix

$I(\alpha, \beta)=\left[\begin{array}{ll}0.000004923 & 0.00000214 \\ 0.00000214 & 0.000000005\end{array}\right] \ldots .(27)$

The above values are computed in (G.R.D) to get the numerical value of Fisher information matrix .

From the variances values and the estimated parameters values, we get the lower and upper values for the estimated parameters which are :

$$
\begin{aligned}
& \hat{\alpha}_{\text {Lower }}=0.0003 \quad ; \quad \hat{\alpha}_{\text {Upper }}=0.009 \\
& ; \quad \hat{\beta}_{\text {Lower }}=0.0127 \\
& \hat{\beta}_{\text {Upper }}=0.013
\end{aligned}
$$

Then computing the numerical values for probability death density function $f(t)$, survival function $s(t)$ and hazard function $h(t)$. 
Table (1) Estimated values for the function $f(t), s(t), h(t)$

\begin{tabular}{|c|c|c|c|c|c|c|}
\hline \multirow{2}{*}{$\begin{array}{c}\text { Failure } \\
\text { Time }\end{array}$} & \multicolumn{2}{|c|}{$f(t)$} & \multicolumn{2}{|c|}{$s(t)$} & \multicolumn{2}{|c|}{$h(t)$} \\
\hline & Lower & Upper & Lower & Upper & Lower & Upper \\
\hline 15 & 0.000598447 & 0.016730928 & 0.002445167 & 0.070363865 & 0.237777275 & 0.244747088 \\
\hline 17 & 0.000598468 & 0.016767939 & 0.002370262 & 0.068267452 & 0.245621277 & 0.252490272 \\
\hline 17 & 0.000598468 & 0.016767939 & 0.002370262 & 0.068267452 & 0.245621277 & 0.252490272 \\
\hline 19 & 0.000598481 & 0.016800725 & 0.002303696 & 0.066400603 & 0.253020669 & 0.259791482 \\
\hline 19 & 0.000598481 & 0.016800725 & 0.002303696 & 0.066400603 & 0.253020669 & 0.259791482 \\
\hline 19 & 0.000598481 & 0.016800725 & 0.002303696 & 0.066400603 & 0.253020669 & 0.259791482 \\
\hline 20 & 0.000598484 & 0.0168158 & 0.002272998 & 0.065538451 & 0.256579154 & 0.263301695 \\
\hline 20 & 0.000598484 & 0.0168158 & 0.002272998 & 0.065538451 & 0.256579154 & 0.263301695 \\
\hline 21 & 0.000598486 & 0.016830108 & 0.002243798 & 0.064717657 & 0.260054355 & 0.266729096 \\
\hline 23 & 0.000598485 & 0.016856687 & 0.002189352 & 0.063185382 & 0.266781441 & 0.273361821 \\
\hline 23 & 0.000598485 & 0.016856687 & 0.002189352 & 0.063185382 & 0.266781441 & 0.273361821 \\
\hline 24 & 0.000598483 & 0.01686907 & 0.002163881 & 0.062467704 & 0.270044655 & 0.276578394 \\
\hline 26 & 0.000598473 & 0.016892249 & 0.002115977 & 0.061116529 & 0.27639412 & 0.282835499 \\
\hline 31 & 0.000598428 & 0.01694255 & 0.002010715 & 0.058140902 & 0.291405013 & 0.297619679 \\
\hline 54 & 0.000597884 & 0.017090042 & 0.001678702 & 0.048695838 & 0.350954887 & 0.356158622 \\
\hline 54 & 0.000597884 & 0.017090042 & 0.001678702 & 0.048695838 & 0.350954887 & 0.356158622 \\
\hline 77 & 0.000596867 & 0.01716451 & 0.001466717 & 0.042617996 & 0.402752637 & 0.40694094 \\
\hline 175 & 0.000587831 & 0.017130481 & 0.000979412 & 0.028509179 & 0.60018805 & 0.600875984 \\
\hline 175 & 0.000587831 & 0.017130481 & 0.000979412 & 0.028509179 & 0.60018805 & 0.600875984 \\
\hline 185 & 0.000586499 & 0.017105647 & 0.000946782 & 0.027557909 & 0.619465405 & 0.620716423 \\
\hline 193 & 0.00058538 & 0.017083452 & 0.000921977 & 0.026834212 & 0.634918257 & 0.636629566 \\
\hline 198 & 0.000584656 & 0.017068556 & 0.000907014 & 0.026397459 & 0.644594967 & 0.646598468 \\
\hline 207 & 0.000583308 & 0.0170398 & 0.000881054 & 0.025639357 & 0.662057138 & 0.664595466 \\
\hline 233 & 0.000579083 & 0.01694318 & 0.000812278 & 0.023628622 & 0.712912444 & 0.717061699 \\
\hline 243 & 0.000577328 & 0.01690085 & 0.000787979 & 0.022917492 & 0.732669403 & 0.737465054 \\
\hline 243 & 0.000577328 & 0.01690085 & 0.000787979 & 0.022917492 & 0.732669403 & 0.737465054 \\
\hline 243 & 0.000577328 & 0.01690085 & 0.000787979 & 0.022917492 & 0.732669403 & 0.737465054 \\
\hline 245 & 0.000576969 & 0.016892049 & 0.000783248 & 0.022778996 & 0.73663572 & 0.74156248 \\
\hline 250 & 0.000576058 & 0.016869563 & 0.000771601 & 0.022437956 & 0.746574332 & 0.751831555 \\
\hline 250 & 0.000576058 & 0.016869563 & 0.000771601 & 0.022437956 & 0.746574332 & 0.751831555 \\
\hline 253 & 0.000575503 & 0.016855743 & 0.000764733 & 0.022236808 & 0.752553552 & 0.758010911 \\
\hline 267 & 0.000572828 & 0.016788034 & 0.000733808 & 0.021330755 & 0.780624243 & 0.787034221 \\
\hline 310 & 0.000563764 & 0.016548134 & 0.000648915 & 0.018840784 & 0.868780116 & 0.878314555 \\
\hline 325 & 0.000560307 & 0.016453567 & 0.000622356 & 0.018061032 & 0.900300611 & 0.91099821 \\
\hline 330 & 0.000559122 & 0.016420837 & 0.00061381 & 0.017810076 & 0.910903011 & 0.921997024 \\
\hline 338 & 0.00055719 & 0.01636723 & 0.000600441 & 0.01741738 & 0.927969307 & 0.939706756 \\
\hline 350 & 0.000554215 & 0.016283999 & 0.000581053 & 0.016847806 & 0.953811246 & 0.96653528 \\
\hline
\end{tabular}

Note that we can make the following comments for the results in the above table :

1- Noting that the values of death density function $f(t)$ were increasing slightly until $(\mathrm{t}=24)$ for lower bound, then the values became decreasing slightly until the end of failure times, but the values of death density function $f(t)$ were increasing slightly until $(\mathrm{t}=77)$ for upper bound, then the values became decreasing slightly until the end of failure times.

2- Noting that the values of survival function $s(t)$ are decreasing gradually with increasing the failure times for the Lung cancer patients in the hospital, that means there is an opposite relationship between failure times and survival function .

3- Noting that the values of hazard function $h(t)$ are increasing gradually with increasing the failure times for the Lung cancer patients in the hospital, that means there is a direct relationship between failure times and hazard function . 


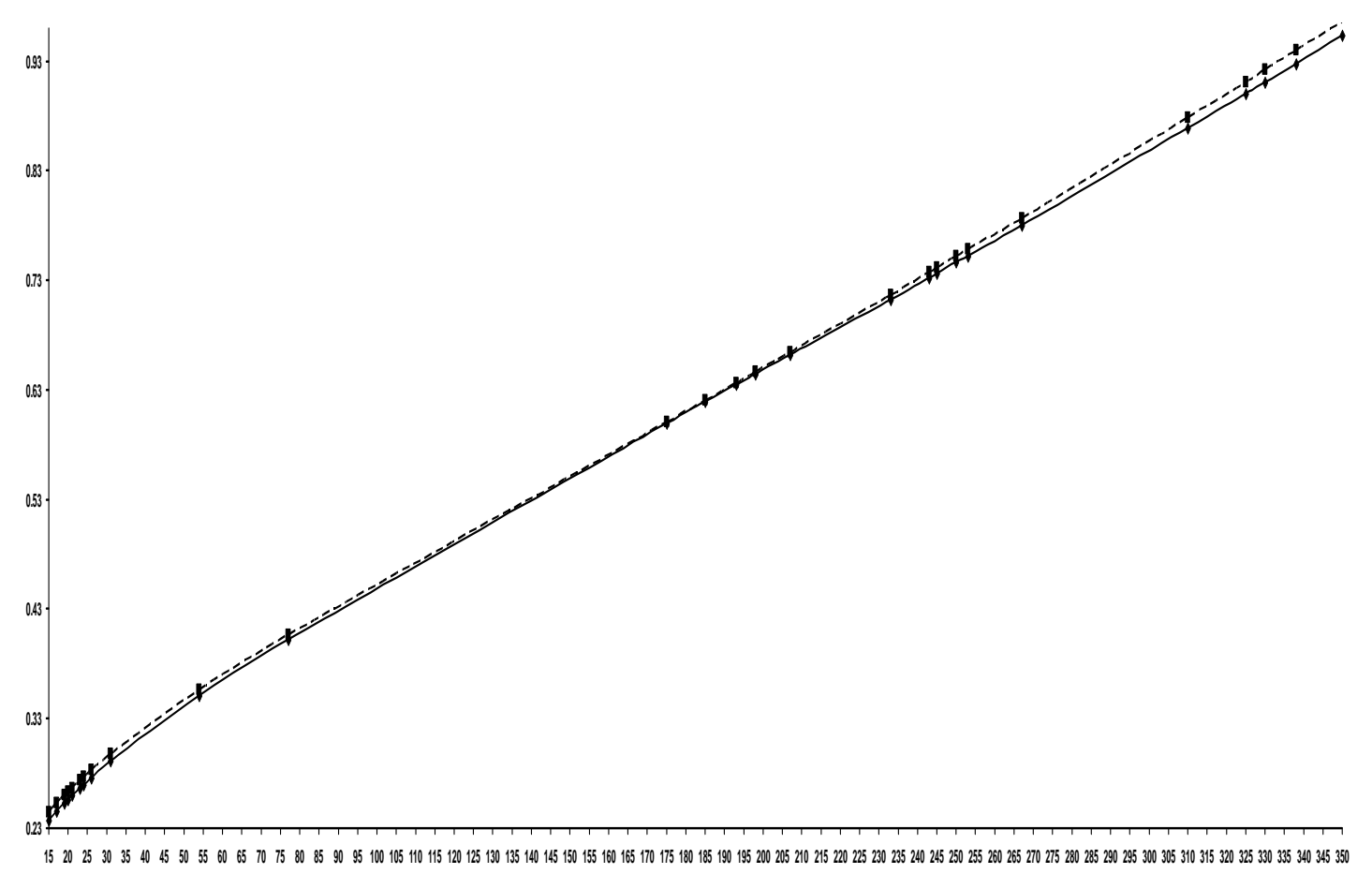

Fig. (3) The relation between lower and upper $h(t)$ with $t$

\section{References:}

1- Amin , E. A., (2011), “ maximum likelihood estimation of the mixed generalized Rayleigh distribution from type I censored samples ", applied mathematical sciences , 5.(56) : 2753-2764 .

2- Das , K. K. and Roy, T. D. ( 2011 ), “ Applicability of length biased weighted generalized Rayleigh distribution", pleagia Research library, 2(4) :320-327.

3- Dey, S. and Dey, T. (2011), “ Rayleigh distribution revisited VIA extension of Jeffreys prior information and a new loss function ", Revstat-Statistical Journal, Vol. 9 ( 3) : 213-226.

4- Kundu , D. and Raqab , M.Z. , (2005) , “ generalized Rayleigh

distribution : Different methods of estimations " , computational statistics and data analysis , 49:187-200 .

5- Mathews J.H. and Fink K.D. (2003) , “ Numerical Method Using MATLAB ", Third Edition , Prentice Hall, USA .

6- Raqab , M. Z. and Kundu , D. , (2006) Burr type $X$ distribution : Revisited , Journal of probability and statistical sciences , 4(2), 179193.

7- Surles , J.G. and Padgett W.J. , (2005) , " some properties of a scaled Burr type X distribution ", Journal of statistical planning and inference, $128: 271-280$. 


$$
\text { * تقدير البقاءع لعينة مراقبة من التوع الأول بالاعتماد على توزيع رالي العام }
$$

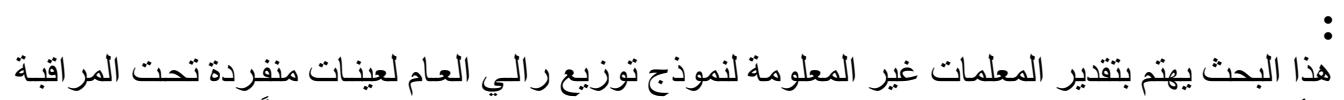

خلاصة : مذا

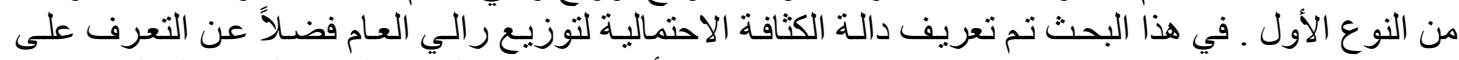

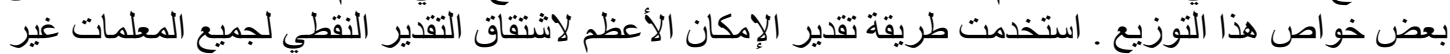

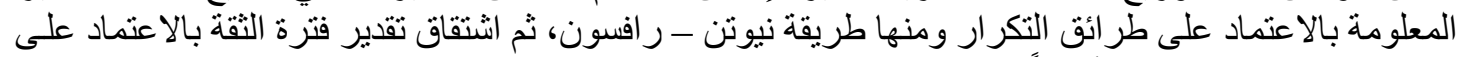

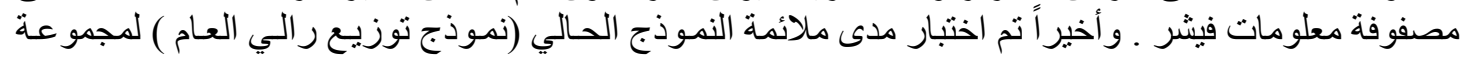

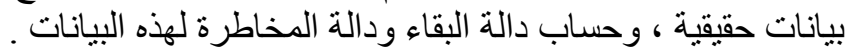

